\title{
Non-Lorentzian chaos and cosmological holography
}

\author{
Arjun Bagchi, ${ }^{1, *}$ Shankhadeep Chakrabortty $\oplus^{2, \dagger}$ Daniel Grumiller ${ }^{3, \dagger}$ Bharathkumar Radhakrishnan $\oplus_{,}^{4,5, \S}$ \\ Max Riegler ${ }^{6,},{ }^{6, \|}$ and Aditya Sinha ${ }^{7,9}$ \\ ${ }^{1}$ Indian Institute of Technology, Kanpur 208016, India \\ ${ }^{2}$ Indian Institute of Technology, Rupnagar, Punjab 140001, India \\ ${ }^{3}$ Institute for Theoretical Physics, TU Wien, Wiedner Hauptstrasse 8-10/136, A-1040 Vienna, Austria \\ ${ }^{4}$ Indian Institute of Science Education and Research, Mohali, Punjab 140306, India \\ ${ }^{5}$ International Centre for Theoretical Sciences, Bangalore, Karnataka 560089, India \\ ${ }^{6}$ Center for the Fundamental Laws of Nature, Harvard University, Cambridge, Massachusetts 02138, USA \\ ${ }^{7}$ Carnegie Mellon University, Pittsburgh, Pennsylvania 15213, USA
}

(Received 24 June 2021; accepted 8 October 2021; published 11 November 2021)

\begin{abstract}
We study chaos in non-Lorentzian field theories, specifically Galilean and Carrollian conformal field theories in two dimensions. In a large central charge limit, we find that the Lyapunov exponent saturates the bound on chaos, conjectured originally for relativistic field theories. We recover the same Lyapunov exponent holographically by a shock-wave calculation in three-dimensional flat space cosmologies, providing further evidence for flat space holography.
\end{abstract}

DOI: 10.1103/PhysRevD.104.L101901

\section{INTRODUCTION}

As aptly put by Edward Lorenz, a dynamical system becomes chaotic when the present determines the future, but the approximate present does not. In other words, the dynamics of the system is strongly sensitive to initial conditions. Colloquially, this is often described by flapping butterfly wings in Brazil causing a tornado in Texas [1]. In this Letter, we discuss aspects of chaos in unconventional quantum field theories (QFTs) and their holographic manifestation in cosmological spacetimes.

The QFTs we consider are non-Lorentzian conformal field theories (CFTs) in two dimensions. We begin our analysis with Galilean CFTs (GCFTs) [2], where the speed of light goes to infinity [3]. GCFTs are natural analogs of relativistic CFTs [4], which appear in the nonrelativistic (NR) limit of all known relativistic conformally invariant QFTs, e.g., massless scalars and fermions in all dimensions, sourceless electrodynamics, and pure non-Abelian gauge theories in four dimensions [5]. GCFTs are governed

\footnotetext{
*abagchi@iitk.ac.in

s.chakrabortty@iitrpr.ac.in

*grumil@hep.itp.tuwien.ac.at

§r.bharathkumar@outlook.com

"mriegler@fas.harvard.edu

asinha5@andrew.cmu.edu
}

Published by the American Physical Society under the terms of the Creative Commons Attribution 4.0 International license. Further distribution of this work must maintain attribution to the author(s) and the published article's title, journal citation, and DOI. Funded by SCOAP . by the Galilean conformal algebra (GCA), which in two dimensions reads [6]

$$
\begin{aligned}
& {\left[L_{n}, L_{m}\right]=(n-m) L_{n+m}+\frac{c_{L}}{12}\left(n^{3}-n\right) \delta_{n+m, 0},} \\
& {\left[L_{n}, M_{m}\right]=(n-m) M_{n+m}+\frac{c_{M}}{12}\left(n^{3}-n\right) \delta_{n+m, 0},} \\
& {\left[M_{n}, M_{m}\right]=0, n, \quad m \in \mathbb{Z} .}
\end{aligned}
$$

Here $c_{L}$ and $c_{M}$ are central charges. We take $c_{L}=0$ and large $c_{M}$, since this is relevant for holographic applications of our results. With methods mirroring recent advances in relativistic two-dimensional (2D) CFTs [7-9] we compute the diagnostics of chaos in GCFTs, specifically, the Lyapunov exponent, which characterizes the divergence of nearby trajectories in phase space.

We are also interested in another class of non-Lorentzian CFTs, viz. Carrollian CFTs (CCFTs). In Carrollian theories, the speed of light goes to zero, the opposite of the Galilean limit [3,10-12]. Interestingly, in $d=2$, the Galilean and Carrollian groups as well as their conformal versions are isomorphic [13]. Hence our answers, constructed for 2D GCFTs, are also valid for 2D CCFTs, albeit with an interchange of the spatial and temporal directions, and correspondingly different physical interpretations.

Finally, we use the holographic correspondence between 3D flat space and 2D CCFTs $[10,13]$ (elaborated on later) to holographically reproduce our field theory answers. The main result of the present Letter is that both on the field theory and the gravity sides the Lyapunov exponents 


$$
\lambda_{L}=\frac{2 \pi}{\beta}
$$

agree and saturate the conjectured bound on chaos [14] ( $\beta$ is the inverse temperature). Since in NR and Carrollian field theories the light cone degenerates (the speed of light tends to infinity and zero, respectively), it is nontrivial that the Lyapunov exponent (2) is finite, nonzero, and coincides with the relativistic result.

\section{GALILEAN CFT BASICS}

We begin with some representation theory aspects of 2D GCFTs. The states of the theory are labeled as [6]

$$
L_{0}|\Phi\rangle=\Delta_{\Phi}|\Phi\rangle, \quad M_{0}|\Phi\rangle=\xi_{\Phi}|\Phi\rangle .
$$

The action of $L_{n}, M_{n}$ for $n>0$ on a state $|\Phi\rangle$ lowers the weight $\Delta_{\Phi}$. Hence in analogy with 2D CFTs, there is a notion of primary states $|\Phi\rangle_{p}$ satisfying

$$
L_{n}|\Phi\rangle_{p}=M_{n}|\Phi\rangle_{p}=0, \quad \forall n>0 .
$$

The rest of the module is built by acting with raising operators $L_{-n}, M_{-n}$ for $n>0$ on a given primary state. Like in relativistic CFTs, there is also a state-operator correspondence $\Phi(0,0) \leftrightarrow|\Phi\rangle$. Below we exclusively use operators.

Chaos in classical systems can be diagnosed via the sensitivity to initial conditions using the Poisson bracket $\{x(t), p(0)\}=\frac{\partial x(t)}{\partial x(0)}$, which can grow as a sum of exponentials in $t$. The exponents therein are called Lyapunov exponents. The analogous quantity for a quantum system in a state that is described by a density matrix $\rho$ is $-\operatorname{Tr}(\rho[x(t), p(0)])[15]$. Random phase cancellations that thermalize this quantity too soon are avoided by considering the square of the commutator. To study chaos of a thermal system in equilibrium at inverse temperature $\beta$ we thus consider the quantity [16-19]

$$
C(t)=-\left\langle[W(t), V(0)]^{2}\right\rangle_{\beta},
$$

which can be written in terms of time-ordered and outof-time-ordered correlation functions (OTOCs). In a generic quantum many-body system the former approach a constant after the relaxation time $\beta$ while the OTOCs start at a large value and decrease over time, resulting in an increase of $C(t)$. Thus, one can use OTOCs to diagnose chaos [20]. To find chaotic behavior in a generic 2D GCFT we study the late time behavior of the following OTOC of pairs of local primary operators in a thermal state

$$
\frac{\left\langle V^{\dagger}(0) W^{\dagger}(t) V(0) W(t)\right\rangle_{\beta}}{\left\langle V^{\dagger}(0) V(0)\right\rangle_{\beta}\left\langle W^{\dagger}(t) W(t)\right\rangle_{\beta}} .
$$

It is evaluated by mapping the 2D GCFT from the plane to a cylinder of radius $\beta$. We work in the approximation $c_{M} / \xi_{n} \gg 1$, where $\xi_{n}=\xi_{V}, \xi_{W}$ are the weights defined in Eq. (3). This allows the use of closed-form expressions of Galilean conformal blocks [21-26].

\section{CHAOTIC CORRELATORS IN GCFT}

We start with a 2D GCFT on a complex plane with coordinates $(u, v)$. Consider two local scalar primary operators $V$ and $W$ with weights $\left(\Delta_{V}, \xi_{V}\right)$ and $\left(\Delta_{W}, \xi_{W}\right)$, respectively. Symmetry restricts the vacuum four-point function [6]

$$
\frac{\left\langle V^{\dagger}\left(u_{1}, v_{1}\right) V\left(u_{2}, v_{2}\right) W^{\dagger}\left(u_{3}, v_{3}\right) W\left(u_{4}, v_{4}\right)\right\rangle}{\left\langle V^{\dagger}\left(u_{1}, v_{1}\right) V\left(u_{2}, v_{2}\right)\right\rangle\left\langle W^{\dagger}\left(u_{3}, v_{3}\right) W\left(u_{4}, v_{4}\right)\right\rangle}=\mathcal{A}
$$

to be a function $\mathcal{A}=\mathcal{A}(\chi, \zeta)$ of the GCFT cross ratios

$$
\chi=\frac{u_{12} u_{34}}{u_{13} u_{24}}, \quad \frac{\zeta}{\chi}=\frac{v_{12}}{u_{12}}+\frac{v_{34}}{u_{34}}-\frac{v_{13}}{u_{13}}-\frac{v_{24}}{u_{24}},
$$

where $u_{n m}:=u_{n}-u_{m}$ and $v_{n m}:=v_{n}-v_{m}$. The amplitude $\mathcal{A}$ is invariant under a map to the (thermal) cylinder $u=\exp \left(\frac{2 \pi}{\beta} \tau\right), v=\frac{2 \pi}{\beta} \sigma \exp \left(\frac{2 \pi}{\beta} \tau\right)$, with a spacelike coordinate $-\infty<\sigma<\infty$ and complexified time $\tau=t_{R}+i t_{E}$, where $t_{R}$ and $t_{E}$ denote real and Euclidean time, and $\tau$ satisfies $\tau \sim \tau+i \beta$.

The OTOC (6) is obtained by an analytic continuation from the Euclidean version of Eq. (7) involving three steps. First, one separates all operators in Euclidean time $t_{E_{n}}=\epsilon_{n}$ such that $\epsilon_{1}<\epsilon_{3}<\epsilon_{2}<\epsilon_{4}$. This preserves the real-time ordering of the operators in Eq. (6). Second, the real time is evolved until the desired values of $t_{R}$. Finally, one takes the $\epsilon_{n}$ to zero. We place $V$ and $V^{\dagger}$ at $\sigma=t_{R}=0$ and $W$ as well as $W^{\dagger}$ at $\sigma=x, t_{R}=t$. This leads to $\left(u_{n}, v_{n}\right)=\left(\exp \left[\frac{2 \pi}{\beta} i \epsilon_{n}\right], 0\right)$ for $n \in\{1,2\}$ and $\left(u_{n}, v_{n}\right)=$ $\left(\exp \left[\frac{2 \pi}{\beta}\left(t+i \epsilon_{n}\right)\right], \frac{2 \pi}{\beta} x \exp \left[\frac{2 \pi}{\beta}\left(t+i \epsilon_{n}\right)\right]\right)$ for $n \in\{3,4\}$.

An illuminating choice of operator positions along the thermal circle is to place them in diametrically opposite pairs, i.e., $\epsilon_{2}=\epsilon_{1}+\beta / 2$ and $\epsilon_{4}=\epsilon_{3}+\beta / 2$. We set $\epsilon_{1}=0$ without loss of generality and define the angular displacement of both operator pairs as $\theta=\frac{2 \pi}{\beta} \epsilon_{3}$, which satisfies $0<\theta<\pi$, maintaining the required operator ordering. The cross ratios $\chi$ and $\zeta$ simplify to

$\frac{1}{\chi}=-\sinh ^{2}\left(\frac{\pi t}{\beta}+\frac{i \theta}{2}\right), \quad \frac{\zeta}{\chi}=-\frac{2 \pi x}{\beta} \operatorname{coth}\left(\frac{\pi t}{\beta}+\frac{i \theta}{2}\right)$.

The first equality in Eq. (8) shows that the cross ratio $\chi$ encircles counterclockwise the point $\chi=1$, which will turn out to be a branch point of the amplitude $\mathcal{A}$. The variable $\zeta$ follows a closed contour that does not enclose singularities or branch cuts of $\mathcal{A}$. 


\section{GCA BLOCKS AND REGGE LIMITS}

For large values of $c_{M} / \xi_{n}$ the amplitude $\mathcal{A}(\chi, \zeta)$ given by Eq. (7) can be written in terms of $s$-channel global GCA blocks [21,22] and three-point coefficients $C_{n m}^{p}$

$$
\begin{aligned}
\mathcal{A}(\chi, \zeta)= & \sum_{p} C_{V V}^{p} C_{W W}^{p} \mathfrak{g}_{W W}^{V V}(p \mid \chi, \zeta), \text { with } \\
\mathfrak{g}_{W W}^{V V}= & 4^{\Delta_{p}-1}(1-\chi)^{\frac{1}{3}\left(\Delta_{V}+\Delta_{W}-\frac{3}{2}\right)} \chi^{\Delta_{p}+\frac{4}{3}\left(\Delta_{W}-2 \Delta_{V}\right)} \\
& \times e^{\frac{\xi}{\chi\left(\frac{4}{3}\left(2 \xi_{V}-\xi_{W}\right)-\frac{\xi_{p}}{\sqrt{1-\chi}}\right)+\frac{\xi(\xi \xi}{\left(\xi_{W}+\xi_{W}\right)}}(1+\chi)^{2-2 \Delta_{p}}}(1+\sqrt{1-\chi})^{2-}
\end{aligned}
$$

Note $\mathfrak{g}_{W W}^{V V}(p \mid \chi, \zeta)$ exhibits a branch cut along $\chi \in(1, \infty)$.

For large, negative values of $t$ the contribution from the identity channel $(p=0)$ dominates since $\chi \approx 0$ and $\zeta \approx 0$. At late times $t \gg \beta$ one has again $\chi \approx 0$ and $\zeta \approx 0$. However, $\chi$ crosses the branch cut along $\chi \in(1, \infty)$ leading to a nontrivial monodromy of $\mathfrak{g}_{W W}^{V V}(p \mid \chi, \zeta)$. This process is equivalent to taking the GCA Regge limit [9]

$$
(1-\chi) \rightarrow e^{2 \pi i}(1-\chi), \quad \chi, \zeta \rightarrow 0, \quad \frac{\zeta}{\chi}=\text { const. }
$$

In the limit (10) the cross ratios $\chi$ and $\zeta$ simplify to

$$
\chi=-\exp \left(-\frac{2 \pi}{\beta} t\right) \epsilon_{12} \epsilon_{34}^{*}, \quad \frac{\zeta}{\chi}=-\frac{2 \pi}{\beta} x
$$

with $\epsilon_{n m}:=i\left[\exp \left(\frac{2 \pi}{\beta} i \epsilon_{n}\right)-\exp \left(\frac{2 \pi}{\beta} i \epsilon_{m}\right)\right]$. The leading behavior of the amplitude (9) is then given by

$$
\frac{\mathfrak{g}_{V, W}^{\text {Regge }}(p \mid x, t)}{N(p, V, W)}=e^{-\frac{2 \pi}{\beta} t\left(2-\Delta_{p}^{\prime}\right)-\frac{2 \pi}{\beta} x \xi_{p}^{\prime}}\left(\epsilon_{12} \epsilon_{34}^{*}\right)^{2-\Delta_{p}^{\prime}} .
$$

where $N(p, V, W)=16^{\Delta_{p}-1} \exp \left(\frac{\pi i}{3}\left[2\left(\Delta_{V}+\Delta_{W}\right)-3\right]\right)$ and $\Delta_{p}^{\prime}=\Delta_{p}-\frac{4}{3}\left(\Delta_{W}-2 \Delta_{V}\right)$, and similarly for $\xi_{p}^{\prime}$. Equation (12) is our first key result.

\section{LYAPUNOV EXPONENT IN GCFT}

For large $c_{M} / \xi_{n}$ and $t \gg \beta$, the OTOC (7) is dominated by the identity block [25]

$$
\mathcal{A}_{\mathbb{1}} \sim 1+\frac{2}{c_{M}}\left(\Delta_{V} \xi_{W}+\Delta_{W} \xi_{V}+\xi_{V} \xi_{W} \zeta \partial_{\chi}\right) \mathcal{F}(\chi),
$$

where $\mathcal{F}(\chi)=\chi^{2}{ }_{2} F_{1}(2,2,4, \chi)$. Applying the limit, we find

$$
\begin{aligned}
\mathcal{A}_{\mathbb{1}}(x, t) & \sim 1+h(x) \exp \left(\frac{2 \pi}{\beta}\left(t-t_{*}\right)\right), \quad \text { with } \\
h(x) & =\frac{48 \pi i}{\epsilon_{12} \epsilon_{34}^{*}}\left[\left(\Delta_{V} \xi_{W}+\Delta_{W} \xi_{V}\right)+\xi_{V} \xi_{W} \frac{2 \pi}{\beta} x\right] .
\end{aligned}
$$

We define the scrambling time $t_{*}$ in Eq. (14) as the timescale where the global block expansion fails [27]:

$$
t_{*} \sim \frac{\beta}{2 \pi} \log c_{M} .
$$

From the exponential behavior of the OTOC (14) we read off the main result of our field theory calculations, the Lyapunov exponent for 2D GCFTs

$$
\lambda_{L}=\frac{2 \pi}{\beta} .
$$

We conclude the GCFT discussion with a few technical remarks. Crossing the branch cut and passing to the second sheet of $\mathcal{A}$ is essential for obtaining chaotic behavior. The sign in front of $h(x)$ in Eq. (14) ensures that the magnitude of $\mathcal{A}_{\mathbb{1}}$ decreases in time for any choice of $\epsilon_{n}$ consistent with the real-time ordering of operators in Eq. (6). It does so in an exponential manner, signaling the onset of chaos in a 2D GCFT.

To obtain the corresponding Carrollian results, one simply replaces $t \leftrightarrow x$. Thus, in stark contrast to CFTs, it is not an OTOC that exhibits chaotic behavior in a CCFT but rather an out-of-space-ordered correlation function. The expression for the Lyapunov exponent stays the same as above.

\section{HOLOGRAPHY AND FLAT SPACE COSMOLOGY}

There have been recent efforts generalizing holography beyond AdS/CFT [28] specifically to 3D asymptotically flat spacetimes $[10,13,29-41]$. In asymptotically flat spacetimes, the asymptotic symmetry group at null infinity is the BondiMetzner-Sachs (BMS) group. Following intuition from 3D anti-de Sitter space [42], it has been conjectured [13] that 2D field theory duals of 3D flat space are governed by the 3D BMS algebra, which turns out to be Eq. (1) again, with $c_{L}=0$ and $c_{M}=3 / G$, where $G$ is the $3 \mathrm{D}$ Newton constant [43]. This means we can check our field theory results in a flat space holographic context.

The zero modes of the most general solutions compatible with BMS boundary conditions are flat space cosmologies (FSCs) $[44,45]$. They describe toy model universes with a contracting and an expanding phase given by the metric

$$
d s^{2}=-\frac{d \tau^{2}}{f(\tau)}+f(\tau) d x^{2}+\tau^{2}\left(d \phi-N_{\phi}(\tau) d x\right)^{2},
$$

where $f(\tau)=\tau_{+}^{2}\left(\tau^{2}-\tau_{0}^{2}\right) / \tau^{2}$ and $N_{\phi}(\tau)=\tau_{0} \tau_{+} / \tau^{2}$. FSCs have cosmological horizons at $\tau=\tau_{0}$. The parameters $\tau_{+}=$ $\sqrt{m}$ and $\tau_{0}=|j| / \sqrt{m}$ determine the FSC mass $M=\frac{m}{8 G}$ and angular momentum $J=\frac{j}{4 G}$. In flat holography, FSCs are dual to thermal states in 2D CCFT. Below, inspired by $[14,17,46-48]$, we perform an analysis of a shock wave in the 
FSC geometry to compute the Lyapunov exponent holographically, validating our field theory result (16).

\section{NULL GEODESICS ON FSC BACKGROUNDS}

A general null geodesic moving on the background (17) satisfies

$$
-\frac{\dot{\tau}^{2}}{f(\tau)}+f(\tau) \dot{x}^{2}+\tau^{2}\left(\dot{\phi}-N_{\phi}(\tau) \dot{x}\right)^{2}=0,
$$

where dots denote derivatives with respect to an affine parameter. The first integrals of the geodesic equations

$$
m \dot{x}-j \dot{\phi}=P, \quad \tau^{2} \dot{\phi}-j \dot{x}=L
$$

yield constants of motion $P$ and $L$ corresponding to the Killing vectors $\partial_{x}$ and $\partial_{\phi}$, respectively. For the sake of simplicity, we set $L=0$ [49]. The above equations can be solved to get

$\dot{\tau}= \pm P, \quad \dot{x}=\frac{P \tau^{2}}{\tau_{+}^{2}\left(\tau^{2}-\tau_{0}^{2}\right)}, \quad \dot{\phi}=\frac{P \tau_{0}}{\tau_{+}\left(\tau^{2}-\tau_{0}^{2}\right)}$,

permitting us to express $x$ and $\phi$ as functions of $\tau$,

$$
d x= \pm \frac{d \tau}{f(\tau)}, \quad d \phi= \pm \frac{N_{\phi}(\tau) d \tau}{f(\tau)}
$$

Here the plus sign corresponds to right-moving and counterclockwise rotating rays with respect to $x$ and $\phi$. The separation of comoving spatial coordinates between two events $\left(x_{i}, \tau_{i}, \phi_{i}\right)$ and $\left(x_{j}, \tau_{j}, \phi_{j}\right)$ along a null geodesic is obtained by integrating Eq. (21),

$$
x_{j}-x_{i}= \pm H\left(\tau_{i}, \tau_{j}\right), \quad \phi_{j}-\phi_{i}= \pm G\left(\tau_{i}, \tau_{j}\right),
$$

where $H=\int_{\tau_{i}}^{\tau_{j}} \frac{\tau^{2} d \tau}{\tau_{+}^{2}\left(\tau^{2}-\tau_{0}^{2}\right)}, \quad G=\frac{\tau_{0}}{\tau_{+}} \int_{\tau_{i}}^{\tau_{j}} \frac{d \tau}{\left(\tau^{2}-\tau_{0}^{2}\right)}$.

\section{SPATIAL SHIFTS AND COSMOLOGICAL CHAOS}

We now consider a left-moving, massless probe sent out from past null infinity $\left(\mathscr{I}^{-}\right)$. It crosses the cosmological horizon of the contracting region, adding a tiny amount of energy $\delta M \ll M$ and angular momentum $\delta J \ll J$ to the background geometry. The probe gets reflected at the timelike causal singularity $\tau=0$ [45] and enters the expanding region from the past cosmological horizon. See Fig. 1. An observer $O$ located in the expanding region of the FSC receives this left-moving signal at a time $\tau_{c}$ and at a point $B=\left(x_{1}, \tau_{c}, \phi_{1}\right)$ and eventually sees it moving toward future null infinity $\left(\mathscr{I}^{+}\right)$. Due to the infinite blueshift at the past cosmological horizon the perturbation $(\delta M, \delta J)$ effectively
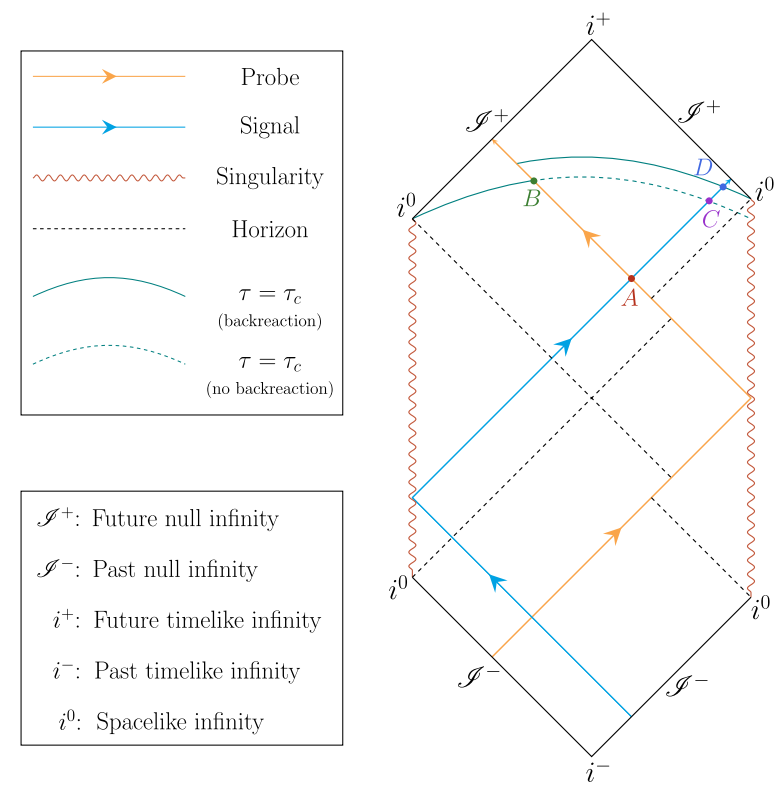

FIG. 1. Penrose diagram depicting the shock-wave FSC geometry. Here the lower (upper) part of the diagram corresponds to a contracting (expanding) universe. The probe is sent out from $\mathscr{I}^{-}$and after a reflection at the singularity emerges from the cosmological horizon where it intersects with the signal very close to the horizon at point $A$ and with the surface $\tau=\tau_{c}$ at point $B$. Without backreaction the signal intersects the surface $\tau=\tau_{c}$ at the point $C$. With backreaction the observer $O^{\prime}$ gets shifted to the point $D$ in order to still be able to receive the signal at $\tau=\tau_{c}$.

causes the formation of a shock-wave geometry in a FSC background. A right-moving null signal crosses the worldline of the probe very close to the horizon at a point $A=\left(x_{\epsilon}, \tau_{\epsilon}, \phi_{\epsilon}\right)$, where $\tau_{\epsilon}=\tau_{0}(1+\epsilon)$ for $1 \gg \epsilon>0$, and intersects the $\tau=\tau_{c}$ surface at $C=\left(x_{2}, \tau_{c}, \phi_{2}\right)$ in the unperturbed geometry where we place a second observer $O^{\prime}$. Due to the backreaction of the probe this observer gets shifted to a different spatial point $\left(\tilde{x}_{2}, \tilde{\phi}_{2}\right)$ on the constant $\tau_{c}$ surface. Below we show that the comoving distance by which the observer $O^{\prime}$ is shifted due to the backreaction of the shock wave grows exponentially with the initial comoving distance between the observers $O$ and $O^{\prime}$, with a numerical prefactor corresponding to the holographic Lyapunov exponent.

Applying Eq. (22) to the spatial separation of comoving coordinates between the events $A$ and $B\left[x_{1}-x_{\epsilon}=\right.$ $\left.-H\left(\tau_{\epsilon}, \tau_{c}\right), \phi_{1}-\phi_{\epsilon}=-G\left(\tau_{\epsilon}, \tau_{c}\right)\right]$ and between the events $A$ and $C\left[x_{2}-x_{\epsilon}=H\left(\tau_{\epsilon}, \tau_{c}\right), \phi_{2}-\phi_{\epsilon}=G\left(\tau_{\epsilon}, \tau_{c}\right)\right]$ yields

$\Delta x=x_{2}-x_{1}=2 H\left(\tau_{\epsilon}, \tau_{c}\right), \quad \Delta \phi=2 G\left(\tau_{\epsilon}, \tau_{c}\right)$.

Solving the integrals (23) for $e^{-\tau_{c} / \tau_{0}} \gg \epsilon>\delta \tau_{0} / \tau_{0}$ and $\tau_{c} \gg \tau_{0}$ gives the leading order behavior of the separation

$$
\Delta x=\frac{\beta}{2 \pi} \log \frac{1}{\epsilon}+\ldots, \quad \Delta \phi=\frac{\mu \beta}{2 \pi} \log \frac{1}{\epsilon}+\ldots,
$$


expressed in terms of inverse Hawking temperature $\beta=2 \pi \tau_{0} / \tau_{+}^{2}$ and angular velocity $\mu=\tau_{+} / \tau_{0}$. To leading order, $\Delta \phi=\mu \Delta x$ and therefore, in what follows we display only $\Delta x$.

A key aspect of Eq. (25) is that the spatial intervals are dominated by the redshift factor $\epsilon$. Holographically, chaos is a consequence of this redshift factor and backreaction effects from the shock wave, which together produce an exponential dependence on the separation $\Delta x$, the prefactor of which is the Lyapunov exponent. To determine this exponent, we consider backreactions.

\section{BACKREACTION AND HOLOGRAPHIC LYAPUNOV EXPONENT}

Backreactions of the shock wave on the FSC background are included by modifying the original FSC parameters $m$ and $j$ to $\tilde{m}=m+\delta m$ and $\tilde{j}=j+\delta j$. As a consequence, the location of the event horizon also changes to $\tau_{0} \rightarrow \tau_{0}+\delta \tau_{0}$, where $\delta \tau_{0}=\frac{\beta}{2 \pi}\left(\mu \delta j-\frac{1}{2} \delta m\right)$. This is the first law of thermodynamics for the FSC geometry,

$$
-T \delta S=\delta M-\mu \delta J, \quad \delta S:=\frac{\delta(\text { area })}{4 G}=\frac{\pi \delta \tau_{0}}{2 G},
$$

featuring a well-documented sign [31,34]. The entropy change $\delta S$ concurs with the Bekenstein-Hawking area law.

Due to the backreaction of the shock wave on the FSC geometry the spatial interval $\Delta x=x_{j}-x_{i}$ changes,

$$
\tilde{x}_{j}-\tilde{x}_{i}= \pm \int_{\tau_{i}}^{\tau_{j}} \frac{d \tau}{\tilde{f}(\tau)}:= \pm \tilde{H}\left(\tau_{i}, \tau_{j}\right) .
$$

Here $\tilde{f}(\tau)=\left.f(\tau)\right|_{m \rightarrow \tilde{m}, j \rightarrow \tilde{j}}$. To linear order in $(\delta m, \delta j)$

$\tilde{H}\left(\tau_{i}, \tau_{j}\right)=H-\delta m \int_{\tau_{i}}^{\tau_{j}} \frac{d \tau}{f(\tau)^{2}}+2 j \delta j \int_{\tau_{i}}^{\tau_{j}} \frac{d \tau}{\tau^{2} f(\tau)^{2}}$.

The above is valid for general probes carrying energy and angular momentum. Probes without angular momentum, i.e., null geodesics with $L=0$, do not change the total angular momentum of the FSC and thus we have $\delta j=0$ and an expanding cosmological horizon in the expanding Universe, as depicted in Fig. 1.

The results (27) and (28) yield the interval

$$
\tilde{x}_{2}-x_{\epsilon}=\tilde{H}\left(\tau_{\epsilon}, \tau_{c}\right)=x_{2}-x_{\epsilon}-\delta m \int_{\tau_{\epsilon}}^{\tau_{c}} \frac{d \tau}{f(\tau)^{2}} .
$$

Shifting $x_{2} \rightarrow \tilde{x}_{2}$ takes care of the necessary shift of $O^{\prime}$ so that the signal is still received at $\tau_{c}$. The crossing point $x_{\epsilon}$ remains the same as before since the correction to this point is subleading in perturbation parameters. Evaluating Eq. (29), the leading order contribution to the spatial shift of the observer,

$$
\tilde{x}_{2}-x_{2} \approx \frac{\beta \delta \tau_{0}}{4 \pi \tau_{0} \epsilon}=\frac{\beta}{4 \pi}(\delta \log S) e^{\frac{2 \pi}{\beta}\left(x_{2}-x_{1}\right)}
$$

contains the entropy change $\delta S$ given in Eq. (26). The results above can also be expressed in a coordinateindependent way in terms of proper distances [50], but we choose a simplified route to match with our field theory answers.

The expression (30) has the same functional form as the field theoretic amplitude (14). To connect these results we first take $\tau_{c}$ to be large, thereby placing the observers $O$ and $O^{\prime}$ close to $\mathscr{I}^{+}$, where the holographic CCFT is encoded. Since Eq. (30) does not explicitly depend on $\tau_{c}$, this expression also holds close to $\mathscr{I}^{+}$. The inverse Hawking temperature $\beta$ can also be interpreted as the periodicity of the Euclidean time circle and hence the (inverse) temperature that an observer in the dual field theory measures. Thus, we find the holographic Lyapunov exponent

$$
\lambda_{L}=\frac{2 \pi}{\beta},
$$

in precise agreement with Eq. (16).

\section{OUTLOOK}

Our results in this Letter moved us into uncharted territories. We generalized 2D CFT results of chaos to non-Lorentzian settings. By studying spatial displacements in cosmological shock-wave solutions, we obtained a holographic Lyapunov exponent matching our field theory results. We shall address further aspects and generalizations of this Letter in [50].

Our results (suitably generalized for higher dimensions) have potentially very diverse applications. They are central to the understanding of chaos in all NR systems in general, including applications in the NR sector of electrodynamics, used e.g., in electrical networks under certain approximations [51] and also in NR quantum chromodynamics [52], which has long been an area of interest for the particle physics community. The $d=2$ Carrollian result is relevant for black hole physics. Black hole event horizons in any dimension carry Carrollian structure [53] and for generic nonextremal black holes, 2D conformal Carrollian structures can be used to understand black hole entropy [54]. We thus expect our results to be useful in the context of thermalization in black holes. Further areas of interest include flat holography and nonrelativistic holography, in the form advocated in [2]. Finally, these symmetries also arise as residual gauge symmetries on the world sheet of tensionless strings $[55,56]$. Chaotic scattering of highly excited strings have been recently studied in a bid to understand chaos in the $S$ matrix of string theory [57]. Since the tensionless sector is also the very-high-energy regime, our results should be significant to high-energy string scattering. 
We close with an important future direction. Chaos is related to the spreading of entanglement [58,59]. Entanglement in Galilean and Carrollian CFTs was studied extensively in $[23,39,60-63]$. We would like to understand how our results can help clarify the spread of entanglement and the speed of entanglement (dubbed "butterfly velocity" in these non-Lorentzian theories.

\section{ACKNOWLEDGMENTS}

We thank Jordan Cotler, Diptarka Das, Shahar Hadar, Elizabeth Himwich, Kristan Jensen, Daniel Kapec, Alexandru Lupsasca, Rohan Poojary, Stefan Prohazka, Jakob Salzer, Joan Simón, Douglas Stanford, and Andy Strominger for delightful scientific discussions and comments. A. B. is partially supported by a Swarnajayanti Fellowship of the Department of Science and Technology
(DST) and the Science and Engineering Research Board (SERB), India. D. G. is supported by the Austrian Science Fund (FWF), Projects No. P 30822, No. P 32581, and No. P 33789. The research of M. R. is supported by the European Union's Horizon 2020 research and innovation program under Marie Skłodowska-Curie Grant Agreement No. 832542 as well as DOE Grant No. de-sc/ 0007870. B. R. was supported in part by the INSPIRE scholarship of the Department of Science and Technology, Government of India, and the Long Term Visiting Students Program of the International Centre for Theoretical Sciences Bangalore. S. C. is partially supported by Institute Scheme for Innovative Research and Development (ISIRD) Grant No. 9-252/2016/ IITRPR/708.
[1] E. Lorenz, Predictibility, in Proceedings of the 139th American Association for the Advancement of Science (AAAS) Meeting, Washington, DC, 1972 (1972), https:// eapsweb.mit.edu/sites/default/files/Butterfly_1972.pdf.

[2] A. Bagchi and R. Gopakumar, Galilean conformal algebras and AdS/CFT, J. High Energy Phys. 07 (2009) 037.

[3] H. Bacry and J. Levy-Leblond, Possible kinematics, J. Math. Phys. (N.Y.) 9, 1605 (1968).

[4] P. Di Francesco, P. Mathieu, and D. Senechal, Conformal Field Theory (Springer, New York, 1997).

[5] A. Bagchi, J. Chakrabortty, and A. Mehra, Galilean field theories and conformal structure, J. High Energy Phys. 04 (2018) 144.

[6] A. Bagchi, R. Gopakumar, I. Mandal, and A. Miwa, GCA in 2d, J. High Energy Phys. 08 (2010) 004.

[7] D. A. Roberts and D. Stanford, Two-Dimensional Conformal Field Theory and the Butterfly Effect, Phys. Rev. Lett. 115, 131603 (2015).

[8] A. L. Fitzpatrick, J. Kaplan, and M. T. Walters, Virasoro conformal blocks and thermality from classical background fields, J. High Energy Phys. 11 (2015) 200.

[9] E. Perlmutter, Bounding the space of holographic CFTs with chaos, J. High Energy Phys. 10 (2016) 069.

[10] A. Bagchi and R. Fareghbal, BMS/GCA Redux: Towards flatspace holography from non-relativistic symmetries, J. High Energy Phys. 10 (2012) 092.

[11] C. Duval, G. W. Gibbons, P. A. Horvathy, and P. M. Zhang, Carroll versus Newton and Galilei: Two dual non-Einsteinian concepts of time, Classical Quantum Gravity 31, 085016 (2014).

[12] A. Bagchi, R. Basu, A. Kakkar, and A. Mehra, Flat holography: Aspects of the dual field theory, J. High Energy Phys. 12 (2016) 147.

[13] A. Bagchi, Correspondence between Asymptotically Flat Spacetimes and Nonrelativistic Conformal Field Theories, Phys. Rev. Lett. 105, 171601 (2010).
[14] J. Maldacena, S. H. Shenker, and D. Stanford, A bound on chaos, J. High Energy Phys. 08 (2016) 106.

[15] A. Larkin and Y. Ovchinnikov, Quasiclassical method in the theory of superconductivity, Sov. Phys. JETP 28, 1200 (1969), http://jetp.ras.ru/cgi-bin/e/index/e/28/6/p1200?a=list.

[16] S. H. Shenker and D. Stanford, Multiple shocks, J. High Energy Phys. 12 (2014) 046.

[17] D. A. Roberts, D. Stanford, and L. Susskind, Localized shocks, J. High Energy Phys. 03 (2015) 051.

[18] S. H. Shenker and D. Stanford, Stringy effects in scrambling, J. High Energy Phys. 05 (2015) 132.

[19] A. Kitaev, Hidden correlations in the Hawking radiation and thermal noise, in Proceedings of the Kavli Institute for Theoretical Physics (KITP), Santa Barbara (2014), http:// online.kitp.ucsb.edu/online/joint98/kitaev/.

[20] F. M. Haehl, R. Loganayagam, P. Narayan, and M. Rangamani, Classification of out-of-time-order correlators, SciPost Phys. 6, 001 (2019).

[21] A. Bagchi, M. Gary, and Zodinmawia, Bondi-Metzner-Sachs bootstrap, Phys. Rev. D 96, 025007 (2017).

[22] A. Bagchi, M. Gary, and Zodinmawia, The nuts and bolts of the BMS bootstrap, Classical Quantum Gravity 34, 174002 (2017).

[23] E. Hijano and C. Rabideau, Holographic entanglement and Poincaré blocks in three-dimensional flat space, J. High Energy Phys. 05 (2018) 068.

[24] E. Hijano, Semi-classical $\mathrm{BMS}_{3}$ blocks and flat holography, J. High Energy Phys. 10 (2018) 044.

[25] W. Merbis and M. Riegler, Geometric actions and flat space holography, J. High Energy Phys. 02 (2020) 125.

[26] M. Ammon, S. Gray, C. Moran, M. Pannier, and K. Wölfl, Semi-classical BMS-blocks from the oscillator construction, J. High Energy Phys. 04 (2021) 155.

[27] Since $c_{M}$ has a physical dimension a more precise definition of scrambling time is $t_{*} \sim \frac{\beta}{2 \pi} \log \frac{c_{M}}{\xi_{n}}$, where $\xi_{n}$ is either $\xi_{V}$ or 
$\xi_{W}$, depending on which weight is bigger. However, since we assume the weights to be finite and $c_{M} / \xi_{n}$ to be large, the quantity $c_{M}$ must be large and hence dominates the scrambling time.

[28] J. M. Maldacena, The large $N$ limit of superconformal field theories and supergravity, Adv. Theor. Math. Phys. 2, 231 (1998).

[29] G. Barnich, A. Gomberoff, and H. A. Gonzalez, The flat limit of three dimensional asymptotically anti-de Sitter spacetimes, Phys. Rev. D 86, 024020 (2012).

[30] A. Bagchi, S. Detournay, and D. Grumiller, Flat-Space Chiral Gravity, Phys. Rev. Lett. 109, 151301 (2012).

[31] A. Bagchi, S. Detournay, R. Fareghbal, and J. Simón, Holography of 3D Flat Cosmological Horizons, Phys. Rev. Lett. 110, 141302 (2013).

[32] G. Barnich, Entropy of three-dimensional asymptotically flat cosmological solutions, J. High Energy Phys. 10 (2012) 095.

[33] G. Barnich, A. Gomberoff, and H. A. González, Threedimensional Bondi-Metzner-Sachs invariant two-dimensional field theories as the flat limit of Liouville theory, Phys. Rev. D 87, 124032 (2013).

[34] A. Bagchi, S. Detournay, D. Grumiller, and J. Simon, Cosmic Evolution from Phase Transition of Three-Dimen sional Flat Space, Phys. Rev. Lett. 111, 181301 (2013).

[35] H. Afshar, A. Bagchi, R. Fareghbal, D. Grumiller, and J. Rosseel, Spin-3 Gravity in Three-Dimensional Flat Space, Phys. Rev. Lett. 111, 121603 (2013).

[36] H. A. Gonzalez, J. Matulich, M. Pino, and R. Troncoso, Asymptotically flat spacetimes in three-dimensional higher spin gravity, J. High Energy Phys. 09 (2013) 016.

[37] R. Fareghbal and A. Naseh, Flat-space energy-momentum tensor from BMS/GCA correspondence, J. High Energy Phys. 03 (2014) 005.

[38] S. Detournay, D. Grumiller, F. Schöller, and J. Simón, Variational principle and one-point functions in threedimensional flat space Einstein gravity, Phys. Rev. D 89, 084061 (2014).

[39] A. Bagchi, R. Basu, D. Grumiller, and M. Riegler, Entanglement Entropy in Galilean Conformal Field Theories and Flat Holography, Phys. Rev. Lett. 114, 111602 (2015).

[40] A. Bagchi, D. Grumiller, and W. Merbis, Stress tensor correlators in three-dimensional gravity, Phys. Rev. D 93, 061502 (2016).

[41] J. Hartong, Holographic Reconstruction of 3D flat spacetime, J. High Energy Phys. 10 (2016) 104.

[42] J. D. Brown and M. Henneaux, Central charges in the canonical realization of asymptotic symmetries: An example from threedimensional gravity, Commun. Math. Phys. 104, 207 (1986).

[43] G. Barnich and G. Compere, Classical central extension for asymptotic symmetries at null infinity in three spacetime dimensions, Classical Quantum Gravity 24, F15 (2007).
[44] L. Cornalba and M.S. Costa, A new cosmological scenario in string theory, Phys. Rev. D 66, 066001 (2002).

[45] L. Cornalba and M.S. Costa, Time dependent orbifolds and string cosmology, Fortschr. Phys. 52, 145 (2004).

[46] S. H. Shenker and D. Stanford, Black holes and the butterfly effect, J. High Energy Phys. 03 (2014) 067.

[47] J. Engelsöy, T. G. Mertens, and H. Verlinde, An investigation of $\mathrm{AdS}_{2}$ backreaction and holography, J. High Energy Phys. 07 (2016) 139.

[48] D. Grumiller and R. McNees, Universal flow equations and chaos bound saturation in 2d dilaton gravity, J. High Energy Phys. 01 (2021) 112.

[49] The case $L \neq 0$ yields essentially the same results and will be addressed in upcoming work [50].

[50] A. Bagchi, S. Chakrabortty, D. Grumiller, B. Radhakrishnan, M. Riegler, and A. Sinha, Chaos and holography in BMS invariant field theories (to be published).

[51] G. Rousseaux, Forty years of Galilean electromagnetism (1973-2013), Eur. Phys. J. Plus 128, 81 (2013).

[52] J. Soto, Overview of non-Relativistic QCD, Eur. Phys. J. A 31, 705 (2007).

[53] L. Donnay and C. Marteau, Carrollian physics at the black hole horizon, Classical Quantum Gravity 36, 165002 (2019).

[54] S. Carlip, Black Hole Entropy from Bondi-Metzner-Sachs Symmetry at the Horizon, Phys. Rev. Lett. 120, 101301 (2018).

[55] A. Bagchi, Tensionless strings and Galilean conformal algebra, J. High Energy Phys. 05 (2013) 141.

[56] A. Bagchi, S. Chakrabortty, and P. Parekh, Tensionless strings from worldsheet symmetries, J. High Energy Phys. 01 (2016) 158.

[57] D. J. Gross and V. Rosenhaus, Chaotic scattering of highly excited strings, J. High Energy Phys. 05 (2021) 048.

[58] M. Mezei and D. Stanford, On entanglement spreading in chaotic systems, J. High Energy Phys. 05 (2017) 065.

[59] M. Mezei, On entanglement spreading from holography, J. High Energy Phys. 05 (2017) 064.

[60] H. Jiang, W. Song, and Q. Wen, Entanglement entropy in flat holography, J. High Energy Phys. 07 (2017) 142.

[61] L. Apolo, H. Jiang, W. Song, and Y. Zhong, Modular Hamiltonians in flat holography and (W)AdS/WCFT, J. High Energy Phys. 09 (2020) 033.

[62] D. Grumiller, P. Parekh, and M. Riegler, Local Quantum Energy Conditions in Non-Lorentz-Invariant Quantum Field Theories, Phys. Rev. Lett. 123, 121602 (2019).

[63] V. Godet and C. Marteau, Gravitation in flat spacetime from entanglement, J. High Energy Phys. 12 (2019) 057. 